\title{
Scaling laws and bounds for the turbulent G.O. Roberts dynamo
}

\author{
A. Tilgner ${ }^{1}$ \\ Institute of Geophysics, University of Göttingen, Friedrich-Hund-Platz 1, \\ 37077 Göttingen, Germany
}

(Dated: 12 September 2018)

Numerical simulations of the G.O. Roberts dynamo are presented. Dynamos both with and without a significant mean field are obtained. Exact bounds are derived for the total energy which conform with the Kolmogorov phenomenology of turbulence. Best fits to numerical data show the same functional dependences as the inequalities obtained from optimum theory.

PACS numbers: 91.25.Cw, 47.65.-d 


\section{INTRODUCTION}

Purely hydrodynamic or magnetohydrodynamic (MHD) flows driven by a volume force in three dimensional periodic boxes have a long history in the study of fundamental properties of turbulence and magnetic field generation. Under the assumption that there are universal features in turbulence independent of the shape of distant boundaries, spatially periodic models should allow us to study those features conveniently. Body forces are frequently used to model the effect of actual boundaries, such as moving propellers, which are too cumbersome to simulate in full. While this problem is mostly rooted in engineering, it has also become of interest in MHD research because of laboratory dynamo experiments in which liquid metal is forced by a moving boundary more easily represented by a volume force in numerical simulations intended to design or reproduce the experiments (as for example in -2$)$. Finally, some mechanisms to drive flows in geo- or astrophysics involve a volume forcing if observed in the appropriate frame of reference, such as precession or tides ${ }^{3}$.

Even the numerically convenient setting of a flow in a periodic box overstrains computational possibilities if Reynolds numbers get too large. Exact analytical solutions for turbulent flows are unknown, but techniques are known to determine at least upper bounds for certain quantities. This approach is frequently called optimum theory. These techniques have been applied to several idealized flows, notably Couette flows and convection in plane layers, and also to MHD problems ( $\underline{\underline{4}} \underline{\underline{6}}$ and references therein). These bounds were obtained by using either a background field technique going back to Hopf $\mathrm{f}^{\underline{7}}$ and Doering and Constantin $\underline{\underline{8}}^{\underline{9}}$, or by using an optimization procedure introduced by Howard $\underline{\underline{9}}$ and later on championed by Busse ${ }^{10}$, mostly in the context of convection. Both of these methods rely on the presence of boundaries where boundary conditions restrict admissible fields, and are not useful in a periodic domain. The results presented here for dynamos draw on the work for non-magnetic flows in periodic boxes by Doering and Foias $\underline{11}$ (see also $\underline{\underline{12}, 13}$ ) who formulate bounds in as general terms as possible. The present paper proceeds by way of example and studies a periodic flow introduced by G.O. Roberts $\frac{14}{}$, but the procedure is applicable to general forcing. The G.O. Roberts flow has served as paradigm for numerous problems $\underline{15}-\underline{19}$ and resembles qualitatively the helical flows generated by convection in rotating systems.

The model which will be considered here is a three dimensional periodic box driven by a force density such that the flow assumed in the G.O. Roberts dynamo is a solution of the 
Navier-Stokes equation with that forcing. In general, this flow is unstable. If magnetic field growth sets in, the most important question is at which amplitude the field will saturate. This issue was already tackled with the tools of weakly nonlinear analysis 20,21 . Far from onset, dimensional or heuristic arguments are necessary 22,23 . Heuristics are always uncertain, and dimensional arguments face the problem that more than one length scale (different periodicity lengths in different directions) or more than one velocity scale (the kinetic velocity and the Alfvèn velocity) may be available to form expressions with certain prescribed units. Optimum or bounding theory on the other hand is rigorous, but has a mixed record regarding its performance in approximating or tightly bounding the quantity of interest. A bound is of course the more interesting the closer it is to the value it bounds. The present paper explores the possibilities of this tool when applied to the G.O. Roberts dynamo.

\section{THE MODEL}

Consider a fluid with density $\rho$, conductivity $\sigma$, kinematic viscosity $\nu$, magnetic permeability $\mu_{0}$ and magnetic diffusivity $\lambda=\mu_{0} \sigma$ moving at a velocity $\boldsymbol{v}(\boldsymbol{r}, t)$ as a function of position $\boldsymbol{r}$ and time $t$. The magnetic field is $\boldsymbol{B}(\boldsymbol{r}, t)$. The flow is driven by the time indepen-

dent force density $\boldsymbol{f}(\boldsymbol{r})$. Periodic boundary conditions are imposed with a periodicity length $h$ along the $z$-axis and $a$ along the $x$ - and $y$-axes. It will later be of interest to apply additional restrictions and to allow mean fields (obtained by averaging over the periodicity volume or over planes) only for certain components. Dimensions are removed from the equations of evolution by using $a$ and $a^{2} / \lambda$ as units of length and time, and by introducing the rescaled variables $\boldsymbol{v} a / \lambda, p a^{2} /(\nu \lambda \rho)$ ( $p$ is the pressure), $\boldsymbol{B} a / \sqrt{\rho \mu_{0} \lambda \nu}$, and $\boldsymbol{f} a^{3} /(\rho \lambda \nu)$. Using the same symbols for the rescaled and for the original variables, the equations of evolution read:

$$
\begin{aligned}
\frac{1}{\mathrm{Pm}}\left(\partial_{t} \boldsymbol{v}+\boldsymbol{v} \cdot \nabla \boldsymbol{v}\right) & =-\nabla p+\nabla^{2} \boldsymbol{v}+(\nabla \times \boldsymbol{B}) \times \boldsymbol{B}+\boldsymbol{f} \\
\partial_{t} \boldsymbol{B}+\nabla \times(\boldsymbol{B} \times \boldsymbol{v}) & =\nabla^{2} \boldsymbol{B} \\
\nabla \cdot \boldsymbol{v}=\nabla \cdot \boldsymbol{B} & =0
\end{aligned}
$$

with $\mathrm{Pm}=\nu / \lambda$. The periodicity enforces $\boldsymbol{v}(\boldsymbol{r}, t)=\boldsymbol{v}(\boldsymbol{r}+\hat{\boldsymbol{x}}, t)=\boldsymbol{v}(\boldsymbol{r}+\hat{\boldsymbol{y}}, t)=\boldsymbol{v}\left(\boldsymbol{r}+l_{z} \hat{\boldsymbol{z}}, t\right)$ with $l_{z}=h / a$ and likewise for $\boldsymbol{B}$. Hats denote unit vectors. 
With $V$ the volume of the periodicity cell, define time averaged densities of magnetic energy $E_{B}$ and dissipation $\epsilon_{B}$ as

$$
E_{B}=\left\langle\frac{1}{V} \int \frac{1}{2} \boldsymbol{B}^{2} d V\right\rangle \quad, \quad \epsilon_{B}=\left\langle\frac{1}{V} \int(\nabla \times \boldsymbol{B})^{2} d V\right\rangle
$$

and time averaged densities of kinetic energy and dissipation as

$$
E_{\mathrm{kin}}=\left\langle\frac{1}{V} \int \frac{1}{2} \boldsymbol{v}^{2} d V\right\rangle \quad, \quad \epsilon_{v}=\left\langle\frac{1}{V} \int\left(\partial_{j} v_{i}\right)\left(\partial_{j} v_{i}\right) d V\right\rangle=\left\langle\frac{1}{V} \int(\nabla \times \boldsymbol{v})^{2} d V\right\rangle,
$$

where $\langle\ldots\rangle$ denotes time average and with the standard index notation in which summation over repeated indices is implied. It will be convenient to use the force amplitude $F$ defined through $\boldsymbol{f}(\boldsymbol{r})=F \boldsymbol{\Phi}(\boldsymbol{r})$, with $F \geq 0$. The shape function $\boldsymbol{\Phi}$ is normalized to $\frac{1}{V} \int|\boldsymbol{\Phi}(\boldsymbol{r})|^{2} d V=1$ and obeys $\nabla \cdot \boldsymbol{\Phi}=0$ (a contribution with non zero divergence to $\boldsymbol{f}$ can always be balanced by the pressure gradient).

A characteristic velocity can be defined from the kinetic energy. Because velocities are given in multiples of $\lambda / a$ in the units chosen here, this characteristic velocity actually is the magnetic Reynolds number Rm defined as

$$
\mathrm{Rm}=\sqrt{2 E_{\mathrm{kin}}}
$$

The hydrodynamic Reynolds number Re is given by

$$
\mathrm{Re}=\mathrm{Rm} / \mathrm{Pm}
$$

The choice of units also leads to a factor Pm in the expression for the total energy $E_{\text {tot }}$ :

$$
E_{\mathrm{tot}}=\frac{1}{\mathrm{Pm}} E_{\mathrm{kin}}+E_{B}
$$

One obtains an energy balance by dotting eq. (1) with $\boldsymbol{v}$, eq. (2) with $\boldsymbol{B}$, integrating over $V$, adding the two equations and using the fact that time derivatives of time averages are zero:

$$
\epsilon_{v}+\epsilon_{B}=F\left\langle\frac{1}{V} \int \boldsymbol{\Phi} \cdot \boldsymbol{v} d V\right\rangle
$$

Equations (113) were solved numerically for a volume force of the form

$$
\boldsymbol{\Phi}=\left(\begin{array}{c}
\sin (2 \pi x) \cos (2 \pi y) \\
-\cos (2 \pi x) \sin (2 \pi y) \\
\sqrt{2} \sin (2 \pi x) \sin (2 \pi y)
\end{array}\right)
$$


in the domain $0 \leq x, y \leq 1,0 \leq z \leq l_{z}$. Velocity and magnetic fields were enforced to be periodic in all three directions. Zero wavenumber modes were allowed for $\boldsymbol{v}$ in the $z$-direction (to conform with the structure of $\boldsymbol{\Phi}$ ) and for $\boldsymbol{B}$ in the $x$ - and $y$-directions (to allow a mean field in the $x, y$-plane).

The results of six suites of simulations will be presented below, three for $l_{z}=1$ and three for $l_{z}=2$. For both $l_{z}$, three series of runs simulated $\mathrm{Pm}=3,1$ and 0.3 for different $F$. The numerical scheme was the same finite difference method implemented on GPUs as used in 19 . Spatial resolutions reached up to $512^{3}$.

\section{BOUNDS}

All the bounds derived below follow from two theorems. The first is the Cauchy-Schwarz inequality stating that

$$
\left|\int g h d V\right|^{2} \leq \int g^{2} d V \cdot \int h^{2} d V
$$

for any two square integrable functions $g$ and $h$, and the second is Poincaré's inequality in the form

$$
\int g^{2} d V \leq l^{2} \int|\nabla g|^{2} d V
$$

where $l^{2}$ is the inverse of the smallest eigenvalue of $-\nabla^{2}$ whose eigenfunction is compatible with the boundary conditions and restrictions imposed on $g^{11}$. We will apply Poincaré's inequality to components of both velocity and magnetic fields and use inverse eigenvalues $l_{v}^{2}$ and $l_{B}^{2}$. Periodicity alone allows spatially constant fields, leading to infinite $l_{v}$ and $l_{B}$. However, we will want to exclude uniform translation and a uniform magnetic field (which does not decay through ohmic diffusion), and $l_{v}$ and $l_{B}$ are determined by the smallest wavevector of the admitted fields. It may be interesting to make different choices for $\boldsymbol{v}$ and $\boldsymbol{B}$ regarding the admissible fields with the largest length scale, as was done at the end of the previous section, so that we keep track of two different lengths, $l_{v}$ and $l_{B}$.

It is now trivial to obtain bounds in which $F$ plays the role of the control parameter. Poincaré's inequality applied to the definitions of energies and dissipation rates yields

$$
\epsilon_{v} \geq \frac{1}{l_{v}^{2}} 2 E_{\mathrm{kin}} \quad, \quad \epsilon_{B} \geq \frac{1}{l_{B}^{2}} 2 E_{B} .
$$

Combined with the Cauchy-Schwarz inequality applied to the eq. (9), this leads to

$$
\frac{\mathrm{Rm}^{2}}{l_{v}^{2}}+\frac{2 E_{B}}{l_{B}^{2}} \leq \epsilon_{v}+\epsilon_{B} \leq F \mathrm{Rm} .
$$


Since all variables in this inequality are positive, it immediately follows that

$$
\mathrm{Rm} \leq F l_{v}^{2}
$$

and from searching the extremum of $E_{B}$ over Rm in eq. (14), that

$$
E_{B} \leq \frac{1}{8}\left(l_{B} l_{v} F\right)^{2}
$$

so that the total energy is bounded from above by

$$
E_{\mathrm{tot}}=\frac{1}{\mathrm{Pm}} E_{\mathrm{kin}}+E_{B} \leq\left(\frac{1}{\mathrm{Pm}} \frac{1}{2} l_{v}^{2}+\frac{1}{8} l_{B}^{2}\right) l_{v}^{2} F^{2} .
$$

This upper bound is of very limited practical interest. The dependence of $E_{\text {tot }}$ in $F^{2}$ suggests that this bound needs to be so large in order to accommodate laminar solutions which will become unstable in an actual time integration at sufficiently large $F$, so that the $E_{\text {tot }}$ obtained in numerical simulations of turbulent flows will be far below this bound. Optimum theory as we currently know it is not able to discriminate between stable and unstable solutions, which is one of its major weaknesses.

For now, we will seek bounds independent of $F$ which relate energies and dissipation rates. One obtains from dotting eq. (1) with $\boldsymbol{f}$ and averaging:

$\frac{1}{\mathrm{Pm}} F\left\langle\frac{1}{V} \int \boldsymbol{\Phi} \cdot\{(\boldsymbol{v} \cdot \nabla) \boldsymbol{v}\} d V\right\rangle=F\left\langle\frac{1}{V} \int \boldsymbol{\Phi} \cdot \nabla^{2} \boldsymbol{v} d V\right\rangle+F\left\langle\frac{1}{V} \int \boldsymbol{\Phi} \cdot\{(\nabla \times \boldsymbol{B}) \times \boldsymbol{B}\} d V\right\rangle+F^{2}$

Two of the integrals appearing in this equation can be manipulated into more convenient forms:

$$
\int \boldsymbol{\Phi} \cdot\{(\boldsymbol{v} \cdot \nabla) \boldsymbol{v}\} d V=-\int v_{i} v_{j} \frac{1}{2}\left(\partial_{i} \Phi_{j}+\partial_{j} \Phi_{i}\right) d V
$$

and

$$
\int \boldsymbol{\Phi} \cdot\{(\nabla \times \boldsymbol{B}) \times \boldsymbol{B}\} d V=-\int B_{i} B_{j} \frac{1}{2}\left(\partial_{i} \Phi_{j}+\partial_{j} \Phi_{i}\right) d V
$$

The tensor $\frac{1}{2}\left(\partial_{i} \Phi_{j}+\partial_{j} \Phi_{i}\right)$ is real and symmetric and therefore has real eigenvalues. Denote by $e$ the largest absolute value of these eigenvalues found anywhere in the volume. One then has

$$
\left|\left\langle\frac{1}{V} \int \boldsymbol{\Phi} \cdot\{(\boldsymbol{v} \cdot \nabla) \boldsymbol{v}\} d V\right\rangle\right| \leq 2 e E_{\mathrm{kin}}
$$

and

$$
\left|\left\langle\frac{1}{V} \int \boldsymbol{\Phi} \cdot\{(\nabla \times \boldsymbol{B}) \times \boldsymbol{B}\} d V\right\rangle\right| \leq 2 e E_{B}
$$


These bounds could be sharpened. In order to improve the first inequality, one could determine the divergence free, periodic field $\boldsymbol{v}$ which maximizes $\int \boldsymbol{\Phi} \cdot\{(\boldsymbol{v} \cdot \nabla) \boldsymbol{v}\} d V$ and obtain a better estimate from there. This procedure requires the solution of a multidimensional linear eigenvalue problem, which is a large numerical effort which does not seem appropriate for this paper. It should also be pointed out that in the context of the G.O. Roberts flow, the first of the above integrals will be grossly overestimated, anyway. It is known from numerical simulations that the velocity field resembles the laminar flow even if the forcing is chosen large enough to create turbulence $\frac{18}{}$. If $\boldsymbol{\Phi}$ is the force field of the Roberts flow of eq. (10) and $\boldsymbol{v}$ the laminar response, then the integral $\int \boldsymbol{\Phi} \cdot\{(\boldsymbol{v} \cdot \nabla) \boldsymbol{v}\} d V$ is strictly zero.

The remaining integral in eq. (18) can be estimated using the Cauchy-Schwarz inequality:

$$
\begin{aligned}
\left\langle\frac{1}{V} \int \boldsymbol{\Phi} \cdot \nabla^{2} \boldsymbol{v} d V\right\rangle & =\left\langle\frac{1}{V} \int \boldsymbol{v} \cdot \nabla^{2} \boldsymbol{\Phi} d V\right\rangle=\frac{1}{V} \int\langle\boldsymbol{v}\rangle \cdot \nabla^{2} \boldsymbol{\Phi} d V \leq \sqrt{\frac{1}{V} \int\langle\boldsymbol{v}\rangle^{2} d V} \sqrt{\frac{1}{V} \int\left|\nabla^{2} \boldsymbol{\Phi}\right|^{2} d V} \\
& \leq \sqrt{\frac{1}{V}\left\langle\int \boldsymbol{v}^{2} d V\right\rangle}\left\|\nabla^{2} \boldsymbol{\Phi}\right\|=\operatorname{Rm}\left\|\nabla^{2} \boldsymbol{\Phi}\right\|
\end{aligned}
$$

with the shorthand notation $\left\|\nabla^{2} \boldsymbol{\Phi}\right\|=\sqrt{\frac{1}{V} \int\left|\nabla^{2} \boldsymbol{\Phi}\right|^{2} d V}$. Inserting these inequalities into eq. (18) leads to

$$
F \leq 2 e E_{\text {tot }}+\left\|\nabla^{2} \boldsymbol{\Phi}\right\| \text { Rm. }
$$

This inequality is finally introduced into $\epsilon_{v}+\epsilon_{B} \leq F \mathrm{Rm}$ (see eq. (14)) to yield

$$
\epsilon_{v}+\epsilon_{B} \leq 2 e E_{\mathrm{tot}} \mathrm{Rm}+\left\|\nabla^{2} \boldsymbol{\Phi}\right\| \mathrm{Rm}^{2}
$$

It can be seen from the definitions of Rm and $E_{\text {tot }}$, eqs. (6) and (8), that $E_{\text {tot }}$ varies as a function of $\mathrm{Rm}$ as $\mathrm{Rm}^{2}$ for $E_{B}=0$ and possibly faster if $E_{B} \neq 0$. The first term on the right hand sides of eqs. (24) and (25) thus exceeds the second term by at least a factor Rm. If we now restrict attention to the limit $\mathrm{Rm} \rightarrow \infty$ and retain only the dominating term in $\mathrm{Rm}$ on the right hand sides in eqs. (24) and (25), we find that asymptotically,

$$
E_{\mathrm{tot}} \geq \frac{1}{2 e} F
$$

and

$$
\epsilon_{v}+\epsilon_{B} \leq 2 e E_{\mathrm{tot}} \mathrm{Rm} .
$$

The first inequality is a lower bound for the total energy in terms of $F$ which complements the upper bound derived above and expresses the scaling expected for turbulent flow. 
The second inequality (27), if used as an equality, is familiar from the Kolmogorov picture of turbulence, in that it states that a dissipation rate is given by an energy multiplied by a large scale Reynolds number. Note that a relation of this type could only be derived for the total energy, not kinetic and magnetic energies separately.

It is always true that $\epsilon_{v}+\epsilon_{B} \geq \epsilon_{B}$, and at large $\mathrm{Rm}$, one expects the dissipation rate to be dominated by ohmic dissipation, so that $\epsilon_{v}+\epsilon_{B} \approx \epsilon_{B}$, and one can transform with little loss eq. (27) into $\epsilon_{B} \leq 2 e E_{\text {tot }} \mathrm{Rm}$. For infinite $\mathrm{Pm}$ (more precisely for $\mathrm{Rm}^{2} / \mathrm{Pm} \rightarrow 0$ ), one has the additional simplification

$$
\epsilon_{B} \leq 2 e E_{B} \mathrm{Rm}
$$

which suggests that in this case, the Kolmogorov phenomenology applies to the magnetic field alone.

The next section will study among others the ratio $\epsilon_{B} / E_{B}$. For infinite $\mathrm{Pm}, \epsilon_{B} / E_{B}$ is evidently bounded from above by $2 e \mathrm{Rm}$. The bound is more complicated in the general case. Inserting $\epsilon_{v} \geq \mathrm{Rm}^{2} / l_{v}^{2}$ into eq. (25) leads to

$$
\frac{\epsilon_{B}}{E_{B}} \leq 2 e \mathrm{Rm}+2 e \frac{E_{\mathrm{kin}}}{E_{B} \mathrm{Pm}} \mathrm{Rm}+\left(\left\|\nabla^{2} \boldsymbol{\Phi}\right\|-\frac{1}{l_{v}^{2}}\right) \frac{\mathrm{Rm}^{2}}{E_{B}}
$$

This expression frequently simplifies, as for instance for the G.O. Roberts flow, because $\left\|\nabla^{2} \boldsymbol{\Phi}\right\|=1 / l_{v}^{2}$ and the last term disappears.

Up to here, this section was kept in general terms independent of a particular choice for $\boldsymbol{\Phi}$. The next section presents numerical simulations of the G.O. Roberts dynamo, which means that $\boldsymbol{\Phi}$ is given by eq. (10), which implies

$$
\left\|\nabla^{2} \boldsymbol{\Phi}\right\|=8 \pi^{2} \quad, \quad e=2 \pi .
$$

The velocity is allowed to have a component independent of $z$ but is required to be periodic with periodicity length 1 in the $x$ - and $y$-directions, so that $1 / l_{v}^{2}=8 \pi^{2}$. The magnetic field on the other hand is allowed to have a mean field along the $x$ - and $y$-directions, but is required to be strictly periodic in the $z$-direction with periodicity length $l_{z}$, so that $1 / l_{B}^{2}=\left(2 \pi / l_{z}\right)^{2}$.

\section{RESULTS}

We start with an overview of the solutions obtained numerically. The G.O. Roberts dynamo is the prototypical $\alpha^{2}$-dynamo with a large mean field for control parameters near 


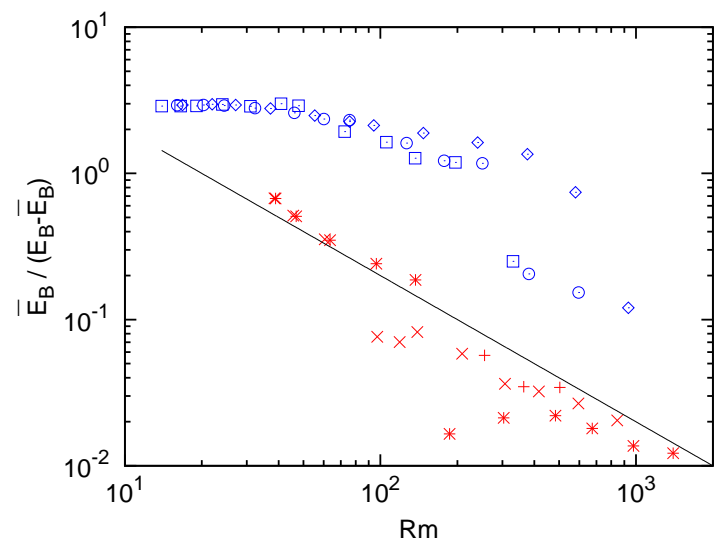

FIG. 1. (Color online) $\bar{E}_{B} /\left(E_{B}-\bar{E}_{B}\right)$, as a function of $\mathrm{Rm}$ for $l_{z}=2$ (blue symbols with an enclosed surface) and $l_{z}=1$ (red symbols built of crosses and stars). For $l_{z}=2, \mathrm{Pm}$ is 0.3 (squares), 1 (circles) and 3 (diamonds). Results for the same Pm are shown for $l_{z}=1: \mathrm{Pm}=0.3$ $(+), 1(\mathrm{x})$ and $3(*)$. The solid line indicates the power law $\mathrm{Rm}^{-1}$.

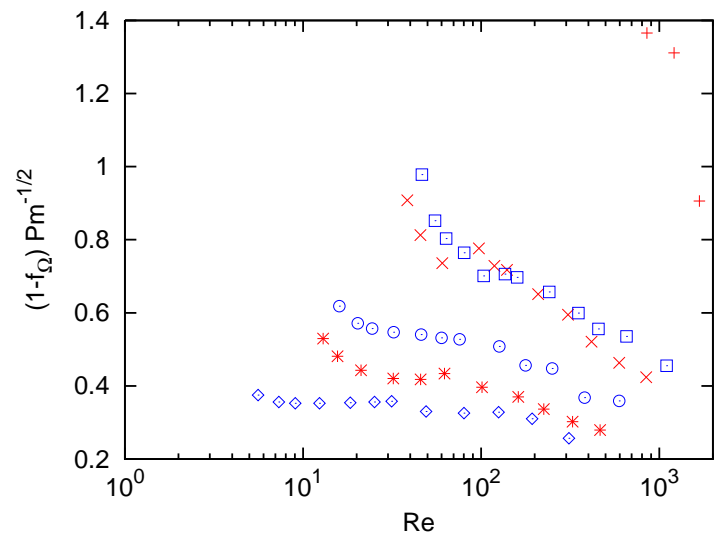

FIG. 2. (Color online) $\left(1-f_{\Omega}\right) \mathrm{Pm}^{-1 / 2}$ as a function of Re with the same symbols as in fig. 1.

a dynamo onset at small Rm. We therefore compute both the total magnetic energy density, $E_{B}$, and the energy density of the mean field,

$$
\bar{E}_{B}=\left\langle\frac{1}{V} A \int d z \frac{1}{2}\left(\frac{1}{A} \int d y \int d x \boldsymbol{B}\right)^{2}\right\rangle
$$

where $A$ is the cross section of the computational volume $V$ in the $x, y$-plane, $A=$ $\int d x \int d y$ 1. The ratio of mean to fluctuating energies, $\bar{E}_{B} /\left(E_{B}-\bar{E}_{B}\right)$, is shown as a function of Rm in figure 1. The computations with the better separation of scales $\left(l_{z}=2\right)$ show a magnetic field dominated by the mean field at low Rm. The contribution of the mean field to the total field suddenly decreases at an Rm between a few hundred and 1000 . 
Beyond that sudden drop, $\bar{E}_{B} /\left(E_{B}-\bar{E}_{B}\right)$ decreases approximately as $1 / \mathrm{Rm}$, whereas the runs with the worse separation of scales $\left(l_{z}=1\right)$ always have $\bar{E}_{B} /\left(E_{B}-\bar{E}_{B}\right)<1$ and show a decrease of this ratio roughly in $1 / \mathrm{Rm}$. A similar abrupt drop as in the case $l_{z}=2$ was observed in convection driven dynamos ${ }^{24}$ where this drop signaled a transition from dynamos generating a mean magnetic field to dynamos which do not generate a mean field and in which all observed mean fields are merely statistical fluctuations. In the convection dynamos, the transition occurred at even smaller Rm than observed here for the G.O. Roberts dynamo. A dramatic decrease of mean field generation at large Rm was already noticed in 18 and could be linked to a transition from a large scale to a small scale dynamo. While the disappearence of the mean field is observed in the dynamos with columnar flow structures mentioned above, it does not appear in less organized turbulent flows ${ }^{25}$. For the purpose of the present paper, it suffices to note that the simulations discussed here contain cases of fields dominated by a mean field as well as cases with a small mean field.

Another result relevant to the discussion below is the ratio of magnetic and total dissipation rate,

$$
f_{\Omega}=\frac{\epsilon_{B}}{\epsilon_{B}+\epsilon_{v}}
$$

This ratio has received much attention in recent years, partly due to an attempt to theoretically predict saturation field strengths in convection dynamos ${ }^{26}$. $f_{\Omega}$ is zero for Rm below the onset of dynamo action and tends to 1 for Rm tending to infinity. Brandenburg 27 found that $\epsilon_{v} /\left(\epsilon_{B}+\epsilon_{v}\right)=1-f_{\Omega}$ scales as $\mathrm{Pm}^{1 / 2}$, and later 28 that $\epsilon_{v} / \epsilon_{B} \propto \mathrm{Pm}^{0.6}$. Finally, a dependence of the exponent on helicity was discovered in ref. ${ }^{29}$. Figure 2 plots $\left(1-f_{\Omega}\right) \mathrm{Pm}^{-1 / 2}$. The factor $\mathrm{Pm}^{-1 / 2}$ obviously has not removed the $\mathrm{Pm}$-dependence from the graph. In fact, there is no recognizable scaling for $f_{\Omega}$ in the present data collection. The point of interest for the discussion below is that $0.07<f_{\Omega}<0.8$ for all points in fig. 2 ,

The most natural global quantity to investigate regarding its scaling behavior is the total energy $E_{\text {tot }}$ as function of the driving force $F$, as shown in fig. 3. The bounding theory of the previous section provides us with an upper and a lower bound on $E_{\text {tot }}$. According to (24) and for $\mathrm{Rm} \rightarrow \infty$, one has for the G.O. Roberts flow $E_{\text {tot }} \geq F /(4 \pi)$, and eq. (17) yields $E_{\text {tot }} \leq F^{2}\left(\frac{1}{\mathrm{Pm}}+\frac{1}{2} l_{z}^{2}\right) /\left(128 \pi^{4}\right)$. As mentioned above, the second bound is required by laminar solutions and can only be of interest for small $F$. The scaling for large $F$ suggested by the first bound can also be inferred from heuristic arguments: The energy injected into, and dissipated by, the flow is in order of magnitude $F U$ with $U$ a characteristic flow velocity, 


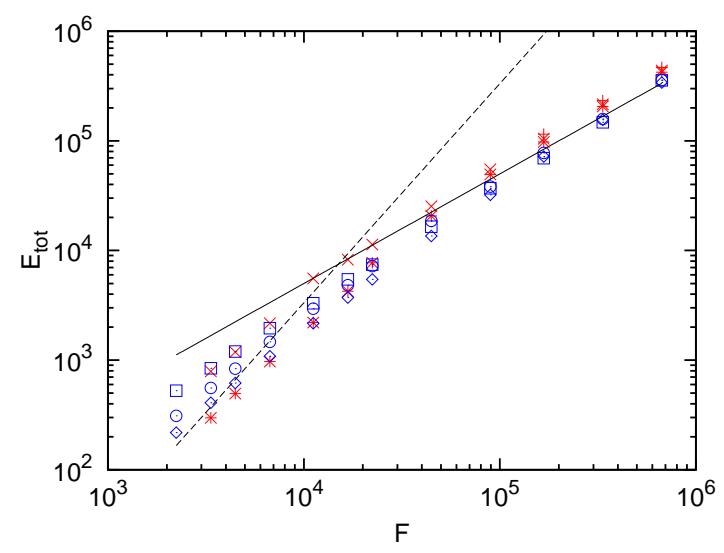

FIG. 3. (Color online) Total energy as a function of the driving force $F$ with the same symbols as in fig. 1. The continuous line shows the function $F / 2$, the dashed line is given by $F^{2} /\left(3 \times 10^{4}\right)$.

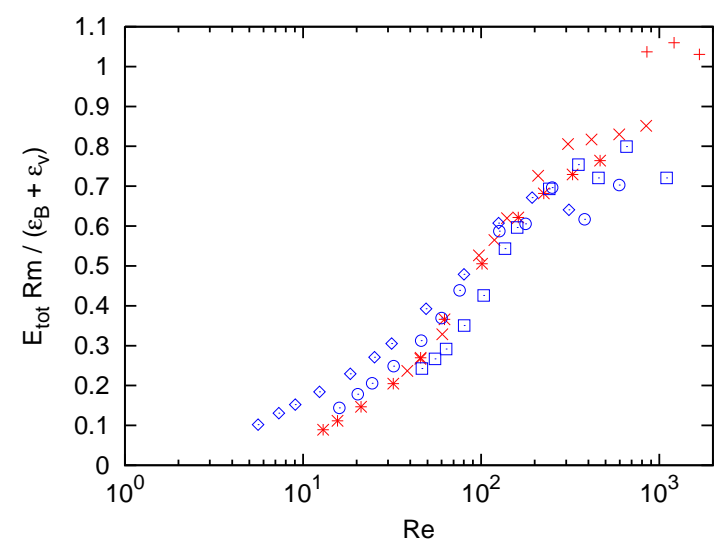

FIG. 4. (Color online) $E_{\text {tot }} \mathrm{Rm} /\left(\epsilon_{B}+\epsilon_{v}\right)$ as a function of Re with the same symbols as in fig. 1.

whereas the total energy dissipation is within the Kolmogorov phenomenology proportional to $E_{\text {tot }} U / L$, so that $E_{\text {tot }} \propto F L$ with $L$ an integral length scale and the proportionality factor is of course left undetermined. This scaling matches the lower bound in eq. (24). The bounding theory provides us with prefactors. In the high Rm limit, this prefactor differs by a factor $2 \pi$ from the prefactor obtained from the fit shown in fig. 3. The exponent on the other hand is close to the one found in the actual simulations, in as far as one accepts exponents deduced from the limited set of data in fig. 3. The upper and lower bounds represent the actual scalings in separate intervals of $F$, because one is based on laminar solutions, the other is not.

The upper bounds for the energy dissipation are not infested by laminar unstable solu- 


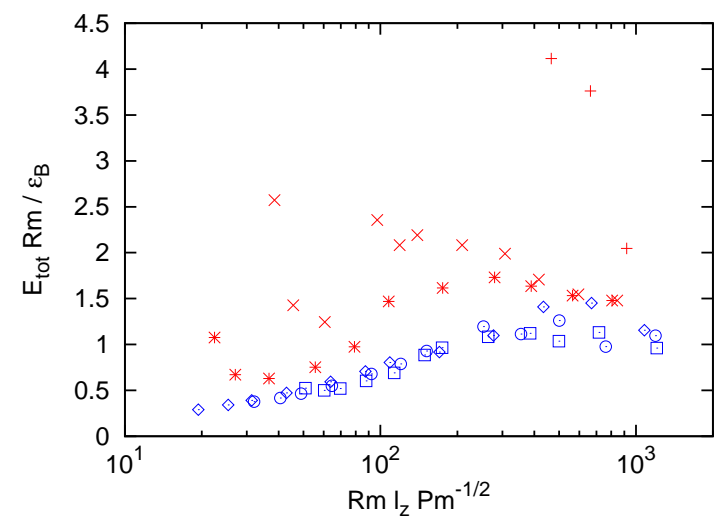

FIG. 5. (Color online) $E_{\text {tot }} \mathrm{Rm} / \epsilon_{B}$ as a function of $\mathrm{Rm}_{z} \mathrm{Pm}^{-1 / 2}$ with the same symbols as in fig. 1. The factor $l_{z} \mathrm{Pm}^{-1 / 2}$ is introduced on the $x$-axis for a better collapse of the data points and a more compact graphical representation, but it has no known physical significance.

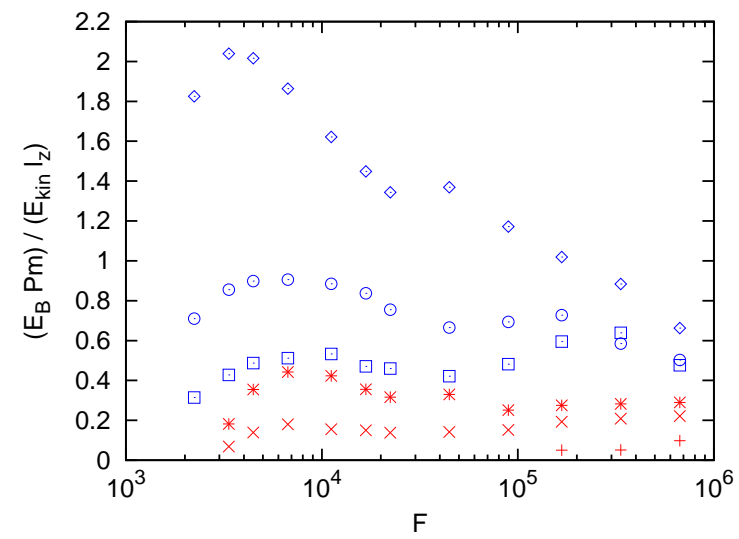

FIG. 6. (Color online) $E_{B} \mathrm{Pm} /\left(E_{\mathrm{kin}} l_{z}\right)$ as a function of $F$ with the same symbols as in fig. 1 .

tions. For large Rm (so that the term $8 \pi^{2} \mathrm{Rm}^{2}$ is negligible in eq. (25)) one has

$$
\epsilon_{v}+\epsilon_{B} \leq 4 \pi E_{\text {tot }} \mathrm{Rm} .
$$

The factor $4 \pi$ is again an order of magnitude larger than the factor found in the simulations at the highest Rm, as seen in fig. 4. However, the important point about this bound is, in analogy with the non-magnetic case ${ }^{11}$, that it provides a rigorous underpinning to the Kolmogorov phenomenology as long as it is applied to total energy and dissipation in the form $\epsilon_{v}+\epsilon_{B} \propto E_{\text {tot }} U / L$. The bounding theory does not in general provide support for any extensions of this phenomenology in which magnetic and kinetic energies are split and which provides us with a scaling for the magnetic field amplitude. Some simplification is achieved 
at large $\mathrm{Rm}$ in which case $\epsilon_{B} \gg \epsilon_{v}$ and eq. (25) simplifies to

$$
\epsilon_{B} \leq 4 \pi E_{\text {tot }} \mathrm{Rm}
$$

Figure 5 verifies relation (34). A regime asymptotic in $\mathrm{Rm}$ of the form $E_{\mathrm{tot}} \mathrm{Rm} / \epsilon_{B} \rightarrow$ const cannot be discernible yet because $\epsilon_{B}$ is not much larger than $\epsilon_{v}$ in any of these simulations. Note the appearance of $E_{\text {tot }}$ instead of $E_{\text {kin }} / \mathrm{Pm}$ on the right hand side of eq. (34). An equation containing only magnetic quantities, at least in the form of a bound, is only obtained in the limit Pm $\rightarrow \infty$ (see eq. (28) ). Eq. (13) always bounds $E_{B}$ in terms of $\epsilon_{B}$ but reflects the scaling pertaining to laminar solutions, so that this bound will be of little practical interest in the turbulent regime.

The distinction between $E_{\text {tot }}$ and $E_{\text {kin }} / \mathrm{Pm}$ on the right hand side of eq. (34) becomes mute if there is equipartition between magnetic and kinetic energies, because then, all energies are proportional to each other. We are therefore led to investigate the ratio $E_{B} \mathrm{Pm} / E_{\text {kin }}$ in fig. 6. This ratio is not perfectly constant as $F$ is varied, but except for the case $l_{z}=2$, $\mathrm{Pm}=3$, it varies around its mean by less than 30\% (and less than a factor of 2 over all). In the range of $\mathrm{Rm}$ in which fig. 5 suggests the validity of a Kolmogorov phenomenology (typically the last three or four points of each series), the ratio of energies varies by at most $20 \%$ around its mean value, while the energies themselves vary by more than an order of magnitude. For the purpose of the scaling implied by eq. (34), $\epsilon_{B} \propto E_{\text {tot }} \mathrm{Rm}$, we have nearly $E_{B} \propto E_{\text {kin }} / \mathrm{Pm} \propto E_{\text {tot }}$ and it cannot be tested whether $E_{\text {tot }}$ on the right hand side of this scaling relation is essential or whether it could be replaced by $E_{B}$.

However, it is clear from fig. [6 that $E_{B} \mathrm{Pm} / E_{\text {kin }}$ depends on $l_{z}$ and $\mathrm{Pm}$, so that there is no strict equipartition between magnetic and kinetic energies, but only a proportionality between the two. The same situation was already found in convection dynamos, in which $E_{B} \propto E_{\text {kin }}$ at high Rm, with a proportionality factor depending on additional control parameters ${ }^{30}$. Ref. ${ }^{18}$ claims to have found equipartition between $E_{B}$ and $E_{\text {kin. However, }}$. this finding was fortuitous because it was obtained for $l_{z}=2$ and $\mathrm{Pm}$ of order 1 . For these parameters, one indeed obtains $E_{B} \approx E_{\text {kin }} / \mathrm{Pm}$, but not at general $l_{z}$ and Pm. From a balance between the Lorentz force term $(\nabla \times \boldsymbol{B}) \times \boldsymbol{B}$ and the advection term $\boldsymbol{v} \cdot \nabla \boldsymbol{v} / \mathrm{Pm}$ and the assumption that $\boldsymbol{B}$ and $\nabla \times \boldsymbol{B}$ are dominated by a mean field which varies on the length scale $l_{z}$ while $\boldsymbol{v}$ varies on the length scale 1 , one obtains $E_{B} \propto l_{z} E_{\mathrm{kin}} / \mathrm{Pm}$. The same result was derived by Brandenburg 22 from an argument based on helicities and the 


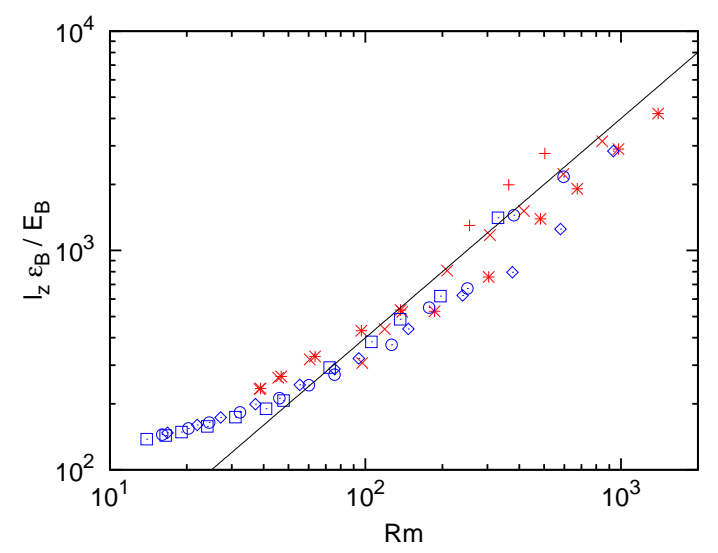

FIG. 7. (Color online) $l_{z} \epsilon_{B} / E_{B}$ as a function of Rm with the same symbols as in fig. 1] The solid line shows the function $4 \mathrm{Rm}$.

assumption of a dynamo field dominated by its mean component. While these arguments do yield a non-trivial prefactor in $E_{B} \propto E_{\text {kin }} / \mathrm{Pm}$, they require a strong mean field which does not exist in all simulations presented here (see fig. 1), so that it comes to no surprise that $E_{B} \mathrm{Pm} /\left(E_{\text {kin }} l_{z}\right)$ does not yield a value independent of all other parameters in fig. 6.

Let us neglect these additional dependences for the moment and assume $E_{\mathrm{kin}} / \mathrm{Pm}$ to be proportional to $E_{B} / l_{z}$. Since $E_{\mathrm{kin}} / \mathrm{Pm}$ is for most points in fig. 6 larger than $E_{B}$, let us approximate $E_{B}+E_{\mathrm{kin}} / \mathrm{Pm} \approx b E_{B} / l_{z}$ with some numerical constant $b$, so that eq. (27) becomes

$$
\epsilon_{v}+\epsilon_{B} \leq b 2 e \frac{E_{B}}{l_{z}} \mathrm{Rm}
$$

For large Rm, one expects $\epsilon_{B} \gg \epsilon_{v}$. The inequality

$$
\epsilon_{B} \leq b 2 e \frac{E_{B}}{l_{z}} \mathrm{Rm}
$$

derived from relation (35) should therefore not be much more inaccurate than relation (35) itself.

Fig. 7 tests eq. (36). At the level of accuracy of double logarithmic plots, the scaling $\epsilon_{B} \propto \frac{E_{B}}{l_{z}} \mathrm{Rm}$ implied by (36) viewed as an equality adequately represents the data cloud at large Rm. It should be stressed that this scaling is not strictly supported by the bounds of the previous section, since some heuristic input was necessary concerning $E_{B} \mathrm{Pm} / E_{\text {kin }}$.

It is of interest to characterize the magnetic field through a time or length scale extracted from its energy and dissipation rate, $\epsilon_{B} / E_{B}$. An initial study on convection dynamos found $\epsilon_{B} / E_{B} \propto \mathrm{Rm}^{31}$. Later investigations yielded smaller exponents ${ }^{24}$. The present computations 
do not reach to high enough $\mathrm{Rm}$ to accurately determine an exponent, but fig. 7 suggests $\epsilon_{B} / E_{B} \propto$ Rm. Eq. (29) applied to the G.O. Roberts flow reads

$$
\frac{\epsilon_{B}}{E_{B}} \leq 4 \pi \mathrm{Rm}\left(1+\frac{E_{\mathrm{kin}}}{E_{B} \mathrm{Pm}}\right) .
$$

If the bracket tends to a constant for $\mathrm{Rm} \rightarrow \infty$, this inequality shows that the exponent $c$ in $\epsilon_{B} / E_{B} \propto \mathrm{Rm}^{c}$ must obey $c \leq 1$.

\section{CONCLUSION}

The G.O. Roberts dynamo is known as a dynamo generating a mean magnetic field, but this study has shown that as Rm is increased, there is a sudden drop in the contribution by the mean field to the total magnetic energy. The same phenomenon was already observed in convection driven dynamos in plane layers, which perhaps is not surprising because both flows consist of helical vortices with parallel axes. However, if this is the essential feature common to both flows, one would expect an analogous behavior in convection driven dynamos in spherical shells. If the analogy is valid, the energy of the axisymmetric modes should suddenly decrease at high Rm in favor of modes with a non zero azimuthal wavenumber.

It is possible to derive several rigorous bounds for the G.O. Roberts dynamo. These concern the total energy, either as a function of the driving force or the total dissipation. The same functional dependences appear in these bounds as in heuristic arguments, but the prefactors in proportionalities are determined in the bounds and differ by about an order of magnitude from prefactors determined from best fits to the numerical data. The prefactors and numerical constants in the bounds presented above could be improved with some numerical effort. The Kolmogorov phenomenology relating total energy to total dissipation is compatible with the bounds. But the optimum theory as presented here does not give any bound of interest for the turbulent regime on magnetic energy alone, unless one is interested in the limit of infinite Pm, or unless one accepts an independent result, extracted from numerical simulations, which states that the ratio of magnetic and kinetic energy is

nearly independent of Rm at large Rm. In these cases, one obtains a phenomenology of the Kolmogorov type for the magnetic field alone. 


\section{ACKNOWLEDGMENTS}

The author acknowledges support from the Helmholtz-alliance "Liquid Metal Technologies".

\section{REFERENCES}

${ }^{1}$ R. Bayliss, C. Forest, M. Nornberg, E. Spence, and P. Terry, "Numerical simulations of current generation and dynamo excitation in a mechanically forced, turbulent flow," Phys. Rev. E 75, 026303 (2007).

${ }^{2}$ K. Reuter, F. Jenko, A. Tilgner, and C. Forest, "Wave-driven dynamo action in spherical magnetohydrodynamic systems," Phys. Rev. E 80, 056304 (2009).

${ }^{3}$ A. Tilgner, "Rotational dynamics of the core," in Treatise on Geophysics, 2nd edition, Vol. 8, edited by G. Schubert (Elsevier, 2015) pp. 183-212.

${ }^{4}$ A. Soward, "Upper bounds for turbulent convective dynamos," Geophys. Astrophys. Fluid Dynam. 15, 317-341 (1980).

${ }^{5}$ R. Kerswell, "Upper bounds on the energy dissipation in turbulent precession," J. Fluid Mech. 321, 335-370 (1996).

${ }^{6}$ T. Alboussière, "Bounds of dissipation on a plane Couette dynamo," Phys. Rev. E 79, $066304(2009)$.

${ }^{7}$ E. Hopf, "Ein allgemeiner Endlichkeitssatz der Hydrodynamik," Mathematische Annalen 117, 764-775 (1941).

${ }^{8}$ C. Doering and P. Constantin, "Energy dissipation in shear driven turbulence," Phys. Rev. Lett. 69, 1648-1651 (1992).

${ }^{9}$ L. Howard, "Bounds on flow quantities," Annual Reviews of Fluid Mechanics 4, 473-494 (1972).

${ }^{10}$ F. Busse, "The optimum theory of turbulence," Advances in Applied Mechanics 18, 77-121 (1978).

${ }^{11}$ C. Doering and C. Foias, "Energy dissipation in body-forced turbulence," J. Fluid Mech. 467, 289-306 (2002).

${ }^{12}$ S. Childress, R. Kerswell, and A. Gilbert, "Bounds on dissipation for Navier-Stokes flow with Kolmogorov forcing," Physica D 158, 105-128 (2001). 
${ }^{13}$ B. Rollin, Y. Dubief, and C. Doering, "Variations on Kolmogorov flow: turbulent energy dissipation and mean flow profiles," J. Fluid Mech. 670, 204-213 (2011).

${ }^{14}$ G. Roberts, "Dynamo action of fluid motions with two-dimensional periodicity," Phil. Trans. Roy. Soc. A 271, 411-454 (1972).

${ }^{15}$ A. Tilgner, "Kinematic dynamos in multiple scale flows," New Journal of Physics 9, 290 (2007).

${ }^{16}$ A. Tilgner, "Dynamo action with wave motion," Phys. Rev. Lett. 100, 128501 (2008).

${ }^{17}$ A. Tilgner and A. Brandenburg, "A growing dynamo from a saturated Roberts flow dynamo," Mon. Not. R. Astron. Soc. 391, 1477-1481 (2008).

${ }^{18}$ Y. Ponty and F. Plunian, "Transition from Large-Scale to Small-Scale Dynamo," Phys. Rev. Lett. 106, 154502 (2011).

${ }^{19}$ V. Tanriverdi and A. Tilgner, "Global fluctuations in magnetohydrodynamic dynamos," New Journal of Physics 13, 033019 (2011).

${ }^{20}$ F. Pétrélis and S. Fauve, "Saturation of the magnetic field above the dynamo threshold," Europhys. J. B 22, 273-276 (2001).

${ }^{21}$ A. Tilgner and F. Busse, "Simulation of the Bifurcation Diagram of the Karlsruhe Dynamo," Magnetohydrodynamics 38, 35-40 (2002).

${ }^{22}$ A. Brandenburg, "The inverse cascade and nonlinear alpha-effect in simulations of isotropic helical hydromagnetic turbulence," Astrophys. J. 550, 824-840 (2001).

${ }^{23}$ F. Pétrélis and S. Fauve, "Scaling laws of turbulent dynamos," C.R. Physique 8, 87-92 (2007).

${ }^{24}$ A. Tilgner, "Magnetic energy dissipation and mean magnetic field generation in planar convection-driven dynamos," Phys. Rev. E 90, 013004 (2014).

${ }^{25}$ S. Candelaresi and A. Brandenburg, "Kinetic helicity needed to drive large-scale dynamo," Phys. Rev. E 87, 043104 (2013).

${ }^{26}$ U. Christensen and J. Aubert, "Scaling properties of convection-driven dynamos in rotating spherical shells and application to planetary magnetic fields," Geophys. J. Int. 166, 97-114 (2006).

${ }^{27}$ A. Brandenburg, "Large-scale dynamos at low magnetic Prandtl numbers," Astrophys. J. 697, 1206-1213 (2009).

${ }^{28}$ A. Brandenburg, "Dissipation in dynamos at low and high magnetic Prandtl numbers," Astron. Nachr. 332, 725-731 (2011). 
${ }^{29}$ A. Brandenburg, "Magnetic Prandtl number dependence of the kinetic-to-magnetic disiipation ratio," Astrophys. J. 791, 12 (2014).

${ }^{30}$ A. Tilgner, "Transitions in rapidly rotating convection dynamos," Phys. Rev. Lett. 109, 248501 (2012).

${ }^{31} \mathrm{U}$. Christensen and A. Tilgner, "Power requirement of the geodynamo from ohmic losses in numerical and laboratory dynamos," Nature 429, 169-171 (2004). 